\title{
Preface: Innovative electrode materials for supercapacitors
}

Portable electrical power sources play increasingly vital roles in our daily lives due to the widespread use of mobile electronic devices and electrical vehicles. Electrochemical capacitors, also referred as supercapacitors (SCs) or ultracapacitors, are an important type of energy storage system with superior advantages of rapid power delivery and recharging compared to other types of energy storage systems. In practice, SCs have played important roles in areas such as engine stop-start systems, regenerative braking of a car, and trams start up. Therefore, SCs have drawn great attention from both academia and industry.

However, SCs, the operation of which rely on the surface adsorption/desorption of ions (EDLC) or surface redox reaction (pseudo capacitance), still suffer from low energy density compared to competitive battery technologies. The improvement of energy density of SCs requires concurrently enhanced cycle life, processing feasibility, safety, and competitive cost. To achieve these targets, the development of SCs should be based on a combination of solid understanding on the electrochemistry of the materials involved, innovative industrial design, and processing technology. SCIENCE CHINA Materials organizes this thematic topic as "Innovative Electrode Materials for Supercapacitors" to demonstrate the most recent advancements and challenges in the field of SCs, especially in the area of electrode materials, the key components of a SC.

Material structure of a SC electrode material is a key factor that affects the device electrochemical performance. A porous structure favors the transport of electrolyte ions and charge, and the porous structure creates rich active sites for electrochemical reactions in SCs. It is not surprising that many efforts have been made to tailor the structure of electrode materials to optimize their porosity and pore size. Metal organic frameworks (MOFs) as a new category of porous materials with tunable pore sizes by adjusting ligands and metal ions used are promising candidates for the electrode materials of SCs. Liu et al. reviewed the recent advancements in
MOFs and their derivatives based electrodes for SCs.

Besides the fundamental science, the development and application of SCs are cost sensitive. Green and sustainable materials made from biomass materials have the potential to reduce material cost. Meanwhile, biomassderived carbon materials show merits of structural diversities, adjustable physical/chemical properties, low price, and environmental friendliness; thus, many researchers have studied these materials. Fan et al. has reviewed biomass-derived carbon materials as electrode materials for SCs.

Various transition metal oxides and hydroxides have been extensively studied as electrode materials for SCs because of their fast and reversible Faradaic process occurring at the interface between the oxides/hydroxides and electrolyte, providing specific capacitance ten times higher than that of EDLC. However, the low electrical conductivity and surface limited reaction of these materials require a rationally designed nanostructure to optimize the electron conduction path and enhance the surface area. Pang et al. provided a summary of the recent progress of hierarchically nanostructured transition metal oxides for SCs. Two research papers about transition metal oxides and hydroxides as electrode materials for SCs are also included. Li et al. reported their results of layered double hydroxides for enhanced pseudocapacitance, and Wang et al. studied $\mathrm{NiCu}$ layered double hydroxide nanosheets with enhanced energy storage performance.

Recently, research on flexible and stretchable electronics is a very hot research topic in the academic community, and this research is expected to be very useful in many new application areas. Nevertheless, finding a suitable power source for flexible electronics is still the bottleneck of this field. Research on the development of flexible SCs has made huge progress in the past decade because of their simpler yet efficient electrochemistry and device configuration than the same for batteries. Zhang et al. summarized the advances in flexible SCs using CNTs and graphene as electrode materials, and a brief de- 
scription of the basic principles for evaluating the performance of flexible SCs is provided in their review. Wu et al. reported their research on all-solid-state micro-SCs with high volumetric capacitance fabricated by mesoporous polypyrrole-based graphene nanosheets with anchored redox polyoxometalate. The device exhibited excellent mechanical flexibility while maintaining good electrochemical performance. In addition, Hou et al. designed a sheath-core flexible electrode by coating $\mathrm{Ni}$ fibers with reduced graphene oxide and $\mathrm{MnO}_{2}$ and assembled a micro-SC device with good flexibility and high performance. Shen et al. also reported their research on flexible wire-shaped SCs with two twisted $\mathrm{NiCo}_{2} \mathrm{O}_{4}$ coated polyvinyl alcohol hydrogel fibers for self-healable and wearable electronic devices.

New design of SCs architecture taking advantage of the high energy density of electrode material such as lithium and sodium is considered a promising approach to enhance the energy density of SCs. Hao et al. reported their results of $\mathrm{T}-\mathrm{Nb}_{2} \mathrm{O}_{4}$ coupled with nitrogen and sulfur codoped graphene as anodes for lithium-ion hybrid SCs, and Yan et al. reported sodium ion hybrid capacitors using nanotube-like hard carbon as the anode material.

The range of electrode materials for SCs in this the- matic issue is diverse. Although different types of electrode materials for SCs have been proposed, in-depth understanding of the electrochemistry involved in the energy storage process is of high importance to facilitate the research and development of SCs towards commercialization. The commercialization will highly rely on the close research collaboration between material design and synthesis, theoretical simulation, multi-scale structure characterization, and rigorous device performance evaluation. Promising materials produced in lab-scale also need to consider their scalability and compatibility with downstream processing technologies in large scale production. This special issue sheds light on the research advancement in the area of SCs towards these important endeavors.

\section{Guest editors}

Professor Hengxing Ji

Department of Materials Science and Engineering, University of Science and Technology of China, China Email: jihengx@ustc.edu.cn Associate Professor Hongxia Wang

School of Chemistry, Physics and Mechanical Engineering, Science and Engineering Faculty, Queensland University of Technology, Australia Email: hx.wang@qut.edu.au 